\title{
On the inverse Compton scattering interpretation of the hard X-ray excesses in galaxy clusters: the case of Ophiuchus
}

\author{
S. Colafrancesco ${ }^{1,2}$ and P. Marchegiani ${ }^{2,3}$
}

\author{
1 ASI-ASDC c/o ESRIN, via G. Galilei snc, 00040 Frascati, Italy \\ e-mail: sergio.colafrancesco@asi.it \\ 2 INAF - Osservatorio Astronomico di Roma via Frascati 33, 00040 Monteporzio, Italy \\ e-mail: cola@mporzio.astro.it \\ 3 Dipartimento di Fisica, Università di Roma La Sapienza, P.le A. Moro 2, Roma, Italy \\ e-mail: marchegiani@mporzio.astro.it
}

Received 13 February 2009 / Accepted 14 April 2009

\begin{abstract}
Context. Populations of high energy electrons can produce hard X-ray (HXR) emission in galaxy clusters by up-scattering CMB photons via the inverse Compton scattering (ICS) mechanism. However, this scenario has various astrophysical consequences. Aims. We discuss here the consequences of the presence of a population of high energy particles for the multi-frequency emissivity of the same clusters and the structure of their atmospheres.

Methods. We derive predictions for the ICS HXR emission in the specific case of the Ophiuchus cluster (for which an interesting combination of observational limits and theoretical scenarios have been presented) for three main scenarios producing high-E electrons: primary cosmic ray model, secondary cosmic rays model and neutralino DM annihilation scenario. We further discuss the predictions of the Warming Ray model for the cluster atmosphere. Under the assumption to fit the HXR emission observed in Ophiuchus, we explore the consequences that these electron populations induce on the cluster atmosphere.

Results. We find that: i) primary electrons can be marginally consistent with the available data provided that the electron spectrum is cutoff at $E \lesssim 30$ and $E \lesssim 90 \mathrm{MeV}$ for electron spectral index values of 3.5 and 4.4, respectively; ii) secondary electron models from pp collisions are strongly inconsistent with the viable gamma-ray limits, cosmic ray protons produce too much heating of the intracluster (IC) gas and their pressure at the cluster center largely exceeds the thermal one; iii) secondary electron models from DM annihilation are also strongly inconsistent with the viable gamma-ray and radio limits, and electrons produce too much heating of the IC gas at the cluster center, unless the neutralino annihilation cross-section is much lower than the proposed value. In that case, however, these models no longer reproduce the HXR excess in Ophiuchus.

Conclusions. We conclude that ICS by secondary electrons from both neutralino DM annihilation and pp collisions cannot be the mechanism responsible for the HXR excess emission; primary electrons are still a marginally viable solution provided that their spectrum has a low-energy cutoff at $E \lesssim 30-90 \mathrm{MeV}$. We also find that diffuse radio emission localized at the cluster center is expected in all these models and requires quite low values of the average magnetic field ( $B \sim 0.1-0.2 \mu \mathrm{G}$ in primary and secondary-pp models; $B \sim 0.055-0.39 \mu \mathrm{G}$ in secondary-DM models) to agree with the available observations. Finally, the WR model (with $B \sim 0.4-2.0 \mu \mathrm{G}$ ) offers, so far, the most accurate description of the cluster in terms of the temperature distribution, heating and pressure and multifrequency spectral energy distribution. Fermi observations of Ophiuchus will provide further constraints to this model.
\end{abstract}

Key words. cosmology: theory - galaxies: clusters: general - galaxies: clusters: individual: Ophiuchus radiation mechanisms: non-thermal

\section{Introduction}

Hard X-ray (HXR) excess emission in galaxy clusters has been observed in the direction of several nearby systems (see Nevalainen et al. 2004) but its origin remains disputed. It has been proposed that this HXR emission is produced by inverse Compton scattering (ICS) of relativistic electrons with the cosmic microwave background (CMB) (Blasi \& Colafrancesco 1999; Atoyan \& Volk 2000; Ensslin \& Biermann 1998; Sarazin 1999; Brunetti 2004; Profumo 2008, see Petrosian et al. 2008 for a recent review), bremsstrahlung emission from a suprathermal electron population (Dogiel et al. 2007, see Petrosian et al. 2008 for a recent review), or a population of PeV electrons that radiates in hard $\mathrm{X}$-rays by synchrotron emission
(Timokhin et al. 2004; Inoue et al. 2005). None of these models has been definitely proven or rejected, so far, because of the lack of instrumental sensitivity (spatial and spectral) of the available experiments operating in this $H X R$ band.

In this context, the Ophiuchus cluster $(z=0.028$, Johnston et al. 1981) has been at the center of an interesting dispute about the combination of new observational evidence of HXR emission and various theoretical considerations about its origin. The Ophiuchus cluster appears to have a high plasma temperature $k T \sim 10 \mathrm{keV}$ (Johnston et al. 1981). Measurements of the IC gas temperature vary from $8.5 \pm 0.5 \mathrm{keV}$ (INTEGRAL; Eckert et al. 2008) to $9.5_{-1.1}^{+1.4} \mathrm{keV}$ (Swift/BAT; Ajello et al. 2009). Watanabe et al. (2004) also found a large $\left(20^{\prime} \times 30^{\prime}\right)$, hot $(k T>13 \mathrm{keV})$ region, 20 arcmin west of the cluster center, which led them 
to conclude that the cluster is not dynamically relaxed, and suggested that it experienced a major merging event in the recent past $(t \lesssim 1 \mathrm{Gyr})$.

Eckert et al. (2008) reported a tentatively resolved $\left(\sim 5^{\prime}\right)$ $\mathrm{X}$-ray source at the cluster center, and the presence of a non-thermal emission tail with a flux of $(10.1 \pm 2.5) \times$ $10^{-12} \mathrm{erg} \mathrm{cm}^{-2} \mathrm{~s}^{-1}$ in the $20-60 \mathrm{keV}$ energy band. These authors interpreted the non-thermal hard X-ray emission as ICS emission from relativistic electrons scattered off the CMB in the intracluster (IC) medium. Suzaku observations of the Ophiuchus cluster by Fujita et al. (2008) have, however, failed to detect the non-thermal component detected by Eckert et al. (2008), although their quoted upper limit of $2.8 \times 10^{-11} \mathrm{erg} \mathrm{cm}^{-2} \mathrm{~s}^{-1}$ in the 20-60 keV energy band is still compatible with the INTEGRAL detection. Ajello et al. (2009) have found, using Swift/BAT spectra (with a IC gas temperature of $k T=9.5 \mathrm{keV}$ ), an upper limit on the Ophiuchus non-thermal X-ray emission in the 20$60 \mathrm{keV}$ band, of $7.2 \times 10^{-12} \mathrm{erg} \mathrm{cm}^{-2} \mathrm{~s}^{-1}$ (90\% c.l.). We note that the INTEGRAL detection and the Swift-BAT upper limit are consistent, at the same $90 \%$ confidence level, in the flux range $(6.1-7.2) \times 10^{-12} \mathrm{erg} \mathrm{cm}^{-2} \mathrm{~s}^{-1}$. This is the flux range in which the HXR excess detected from Ophiuchus is consistent with both Swift-BAT and INTEGRAL observations. In our study of the origin of this HXR excess, we refer to the value $F_{20-60 \mathrm{keV}}=$ $7.2 \times 10^{-12} \mathrm{erg} \mathrm{cm}^{-2} \mathrm{~s}^{-1}$ as the maximum value of its flux and we discuss how our results change by also considering the minimum flux of the HXR, $F_{20-60 \mathrm{keV}}=6.1 \times 10^{-12} \mathrm{erg} \mathrm{cm}^{-2} \mathrm{~s}^{-1}$, which is $\sim 15 \%$ less than the previous maximum value.

The possible presence of an ICS tail of HXR emission in Ophiuchus was previously related to a steep-spectrum radio source MSH 17-203 (also dubbed Cul 1709-231) as a radio mini halo (Johnston et al. 1981), which would thus imply the presence of relativistic electrons, and hence the relative ICS emission emerging from the thermal bremsstrahlung emission in the X-ray band at $E \lesssim 20 \mathrm{keV}$.

However, the high resolution study of Perez-Torres et al. (2008) performed with 240 and $607 \mathrm{MHz}$ GMRT radio observations of the Ophiuchus cluster of galaxies, along with archival 74 and $1400 \mathrm{MHz}$ VLA data, indicates that there is no significant diffuse radio emission in the core of the Ophiuchus, and that the previous measurements of radio flux from the MSH 17-203 source (Slee \& Higgins 1975; Slee 1977) do not refer to the radio halo of the cluster: these authors present new upper limits to the integrated, diffuse non-thermal radio emission from the cluster core. Govoni et al. (2009) pointed out that there is a diffuse radio mini-halo located at the center of the Ophiuchus cluster, with angular size of $\sim 9 \times 12 \mathrm{arcmin}^{2}$; the flux of the mini halo is $\sim 8$ times lower than the old measurement of Johnston et al. (1981), which had a resolution of 80 arcmin (see details in Perez-Torres et al. 2008).

There is no other information on non-thermal emission from Ophiuchus: gamma-ray emission from this cluster has not been detected and therefore the only information that we have directly about the high-E particle population of Ophiuchus is an upper limit obtained by EGRET $F(>100 \mathrm{MeV})=5 \times 10^{-8} \mathrm{~cm}^{-2} \mathrm{~s}^{-1}$ (Reimer et al. 2003).

In this obervational scenario (similar to that of other clusters where an HXR emission detection was claimed) the HXR emission excess from Ophiuchus was interpreted as ICS emission from either a population of primary cosmic ray electrons (Eckert et al. 2008) or secondary electrons produced in neutralino DM annihilation (Profumo 2008). In particular, Profumo (2008) proposed that a combination of three different neutralino DM models $\left[M_{\chi}=81\left(W^{+} W^{-}\right), 40(b \bar{b})\right.$ and $\left.10\left(\tau^{+} \tau^{-}\right) \mathrm{GeV}\right]$ is consistent with all non-thermal emission data for Ophiuchus, from radio to HXR and gamma-rays. The available data on diffuse radio emission in the core of Ophiuchus and the overall analysis of its multi-frequency SED further led Perez-Torres et al. (2008) to conclude that: i) a synchrotron+ICS model from primary cosmic ray electrons is in marginal agreement with the the available data, for a range of magnetic field values $B \sim$ $0.02-0.3 \mu \mathrm{G}$; ii) that a pure neutralino annihilation scenario cannot reproduce both radio and HXR emission, unless extremely low magnetic field values $\left(10^{-2}\right.$ to $\left.10^{-3} \mu \mathrm{G}\right)$ are assumed; iii) a scenario in which synchrotron and ICS arise from PeV electronpositron pairs (via interactions with the $\mathrm{CMB}$ ), can also be excluded, since it predicts a non-thermal soft X-ray emission that largely exceeds the thermal bremsstrahlung emission measured by INTEGRAL.

In this paper, we take a more radical approach to the problem of the HXR emission of Ophiuchus and consider not only the SED properties of synchrotron plus ICS scenarios (from both primary and secondary electrons) but also the physical consequences of the ICS origin of the HXR emission in all models so far viable: primary electron model (Sect. 2.1), secondary electron models from pp collisions (Sect. 2.2) and from DM annihilation (Sect. 2.3) and finally a Warming Ray model (Sect. 3). We discuss our conclusions in Sect. 4.

Throughout the paper, we use a flat, vacuum-dominated cosmological model with $\Omega_{\mathrm{m}}=0.3, \Omega_{\Lambda}=0.7$ and $h=0.7$.

\section{Modeling the electron populations in Ophiuchus}

The spatial distribution of the IC thermal plasma in the Ophiuchus cluster can be represented by

$n_{\mathrm{th}}(r)=n_{\mathrm{th}, 0}\left[1+\left(\frac{r}{r_{\mathrm{c}}}\right)^{2}\right]^{-q_{\mathrm{th}}}$

where $r_{\mathrm{c}}=108 h_{70}^{-1} \mathrm{kpc}$ and $q_{\mathrm{th}}=0.96$ (Watanabe et al. 2004), and $n_{\text {th }, 0}=1.77 \times 10^{-2} h_{70}^{1 / 2} \mathrm{~cm}^{-3}$ (Johnston et al. 1981). The available data for this cluster indicate, to a reasonable extent, an isothermal temperature profile with a temperature $k T \sim 9.9 \mathrm{keV}$ (Watanabe et al. 2004; Ajello et al. 2009); the virial radius of the cluster is $R \sim 1.7 h_{70}^{-1} \mathrm{Mpc}$ (Mohr et al. 1999).

Non-thermal electrons capable of producing the cluster HXR emission by ICS on CMB photons must have energies $E_{\mathrm{e}} \approx$ $0.35 \mathrm{GeV}(E / \mathrm{keV})^{1 / 2}$ in the range $\approx 1.6-2.7 \mathrm{GeV}$, in the case of HXR emission observed in the 20-60 keV range (see, e.g., Colafrancesco et al. 2005).

We discuss, in the following, the predictions of various models for the origin of the high-E electrons: i) primary electron model (PEM) (see, e.g., Colafrancesco et al. 2005 and references therein); ii) secondary electron model produced by protonproton (pp) collisions in the cluster atmosphere (SEM-pp) (see, e.g., Colafrancesco \& Blasi 1998; Blasi \& Colafrancesco 1999; Marchegiani et al. 2007); iii) secondary electron models produced by neutralino Dark Matter annihilation (SEM-DM) (see e.g., Colafrancesco et al. 2006; Profumo 2008).

The electron spectra expected in the previous models are normalized by assuming that the produced ICS HXR emission equals the Swift-BAT/INTEGRAL data.

In addition to the previous models, we also consider a self-consistent warming-ray (WR) electron model (see e.g. Colafrancesco \& Marchegiani 2008; Colafrancesco et al. 2004) in which the cluster atmosphere is heated - in a quasi stationary equilibrium condition between heating and cooling - by the 
interactions of non-thermal cosmic-ray protons with the IC gas. We note that this WR model reproduces the X-ray properties of the thermal IC gas (namely its temperature and density profiles) and, therefore, we use this constraint to predict the cluster ICS HXR emission.

\subsection{Primary electron model (PEM)}

The high-E electron spectrum is usually constrained by using the radio halo synchrotron spectrum that provides direct information about the electron spectral shape (see e.g., Colafrancesco et al. 2005 for a discussion). However, the upper limits to the Ophiuchus radio halo (see Perez-Torres et al. 2008) and the measurement at $1.4 \mathrm{GHz}$ made by Govoni et al. (2009) are insufficient to determine precisely the electron spectrum. Therefore, we choose to adopt here a simple power-law model

$N_{\mathrm{e}}(E, r)=N_{\mathrm{e}, 0}(E / \mathrm{GeV})^{-p} \cdot g(r)$

with $p=3.5$, which corresponds to a radio spectral index of $\alpha_{\mathrm{R}}=1.25$, typical of radio halos in galaxy clusters and consistent with the available radio data on Ophiuchus; we also consider the case with $p=4.4$, which provides $\alpha_{\mathrm{R}}=1.7$, which is, at the $1 \sigma$ level, the maximum value of the spectral index allowed by radio data (see, e.g., Fig. 1). The radial function $g(r)$ should be constrained, in principle, by measuring the radial shape of the ICS emission of the relativistic electrons (the radio measures are not sufficient to constrain this shape; see discussion in Colafrancesco et al. 2005); since the available HXR measures of the Ophiuchus cluster have insufficient spatial resolution, we assume that the radial distribution function of the non-thermal electrons has the same shape as the thermal gas radial distribution (see Eq. (1)). Under such assumption, we consistently assume that the relativistic electrons extend out to the virial radius of the cluster, like the IC plasma.

The value of the normalization of the electron spectrum $N_{\mathrm{e} 0}$ can be derived by reproducing the value of the HXR flux set by the Swift-BAT and INTEGRAL experiments: for the previous spectral index, we obtain the value $N_{\mathrm{e}, 0}=$ $1.1 \times 10^{-10} \mathrm{GeV}^{-1} \mathrm{~cm}^{-3}$ for $p=3.5$, and $N_{\mathrm{e}, 0}=2.1 \times$ $10^{-10} \mathrm{GeV}^{-1} \mathrm{~cm}^{-3}$ for $p=4.4$.

All the previous information can be derived, strictly speaking, for only the range of the electron energies that produce the HXR emission by means of ICS. To obtain information about other energy ranges of the electron spectrum, one must consider other constraints.

The only other constraint that can be placed on the electron spectrum comes from the requirement that the heating rate of the IC gas produced by non-thermal electron Coulomb collisions does not exceed the bremsstrahlung cooling rate of the IC gas. The heating rate produced by an electron with Lorentz factor $\gamma$ and velocity $v=\beta c$ is given by

$-\frac{\mathrm{d} E}{\mathrm{~d} t} \approx K z^{2} Z^{2} \frac{1}{\beta}\left[\ln \frac{2 m_{\mathrm{e}} c^{2} \beta^{2} \gamma^{2}}{I_{\mathrm{p}}}-\beta^{2}\right]$,

where $Z^{2}$ is the (suitably averaged) squared charge of the plasma's nuclei, $K=4 \pi n_{\text {th }} r_{\mathrm{e}}^{2} m_{\mathrm{e}} c^{3}$, with $r_{\mathrm{e}}=\mathrm{e}^{2} / m_{\mathrm{e}} c^{2} \simeq$ $2.82 \mathrm{fm}$, and $I_{\mathrm{p}}=\hbar \omega_{\mathrm{p}}$, where $\omega_{\mathrm{p}}=\left[4 \pi n_{\mathrm{e}} \mathrm{e}^{2} / m_{\mathrm{e}}\right]^{1 / 2}$ is the plasma frequency (see Colafrancesco et al. 2004; Colafrancesco \& Marchegiani 2008).

As a consequence, the heating rate induced by the electrons with the spectrum assumed in Eq. (2) is given by:

$\left.\frac{\mathrm{d} \epsilon}{\mathrm{d} t}\right|_{\mathrm{WR}} \equiv \int_{E_{\min }}^{E_{\max }} N_{\mathrm{e}}(E, r)\left(\frac{\mathrm{d} E}{\mathrm{~d} t}\right) \mathrm{d} E$,
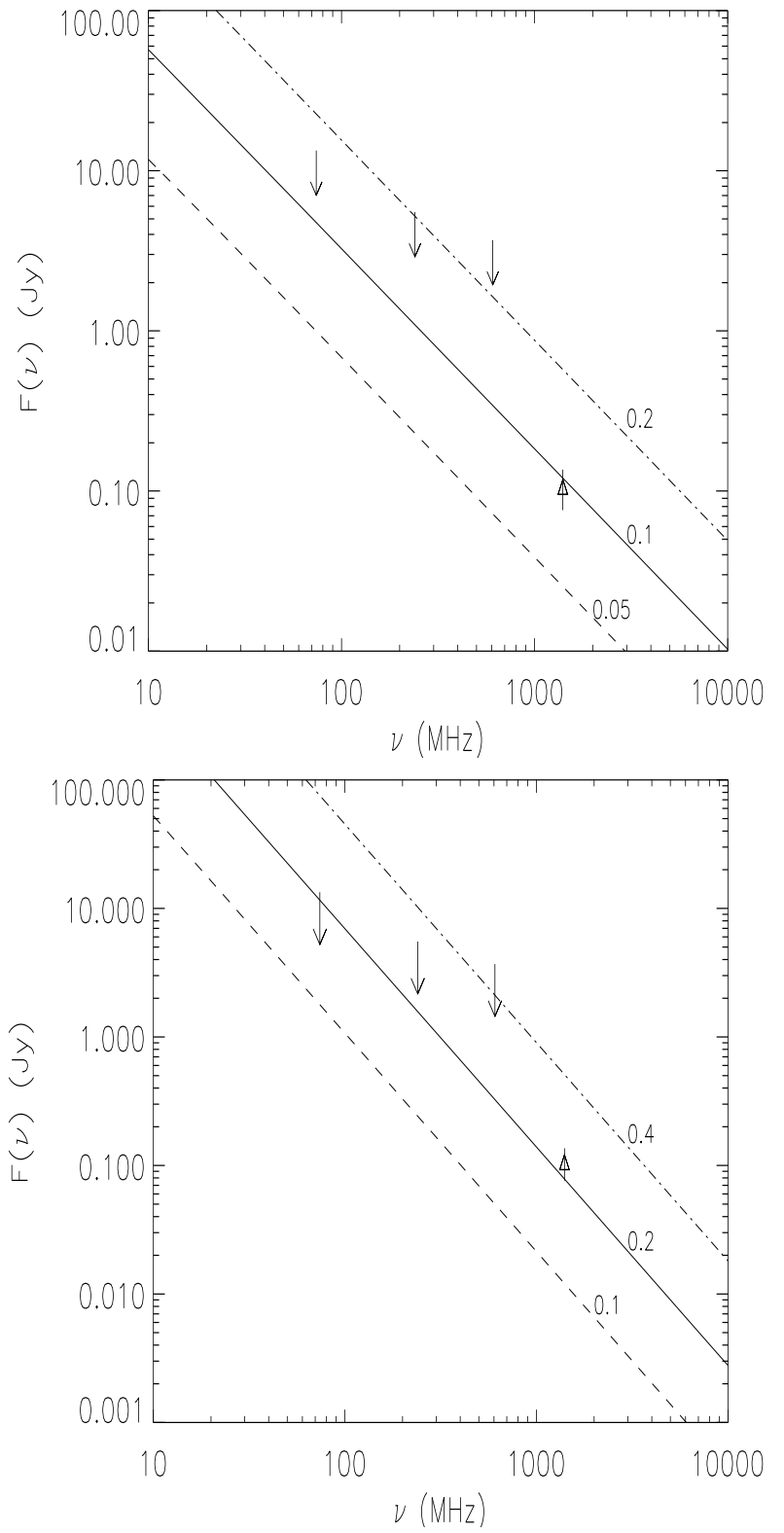

Fig. 1. The diffuse radio-emission spectrum produced by primary electrons with $p=3.5$ (upper panel) and $p=4.4$ (lower panel) is shown for different values of the uniform magnetic field (in units of $\mu \mathrm{G}$ ), as labelled. Data are from Perez-Torres et al. (2008) (upper limits) and Govoni et al. (2009) (point at $1.4 \mathrm{GHz}$ ).

while the cooling rate is given by

$$
\begin{aligned}
\left.\frac{\mathrm{d} \epsilon}{\mathrm{d} t}\right|_{\mathrm{X}} & =a\left[n_{\mathrm{th}}(r)\right]^{2} \sqrt{k T(r, t)} \\
a & =\sqrt{\frac{2^{11} \pi^{3}}{3^{3}}} \frac{\mathrm{e}^{6} \sqrt{m_{\mathrm{e}}}}{h m_{\mathrm{e}}^{2} c^{3}} \bar{z} \\
& \sim 4.8 \times 10^{-24} \bar{z} \frac{1}{\sqrt{\mathrm{keV}}} \frac{\mathrm{erg} \mathrm{cm}}{\mathrm{s}}
\end{aligned}
$$

where $\bar{z}$ is an average charge of the IC plasma (we have approximated here the Gaunt factor $\bar{G}$ by unity).

The two expressions in Eqs. (4) and (5) equal (to the value $\left.\mathrm{d} \epsilon / \mathrm{d} t \sim 4.7 \times 10^{-27} \mathrm{erg} \mathrm{cm}^{-3} \mathrm{~s}^{-1}\right)$ for the energy $E_{\min } \sim 33 \mathrm{MeV}$ for $p=3.5$, and for $E_{\min } \sim 91 \mathrm{MeV}$ for $p=4.4$; we therefore, 
assume that these energy values represent the minimum energy of the primary electron spectra. As for the maximum electron energy, we can safely choose $E_{\max } \rightarrow \infty$ since its specific value is irrelevant for the assumed spectral index.

Based on these assumptions, it is possible to calculate the overall radiation emission by the various emission mechanisms in different frequency ranges.

The synchrotron emission spectrum produced at radio frequencies by primary electrons is shown in Fig. 1 for different values of the magnetic field (the B-field has been assumed to be constant in the emission region, and this corresponds, approximately, to consider a volume averaged value of the magnetic field). From the radio emission, we can derive a value of the average magnetic field of $\sim 0.1 \mu \mathrm{G}$ for $p=3.5$ and $\sim 0.2 \mu \mathrm{G}$ for $p=4.4$, which is in agreement with that derived by Ajello et al. (2009) and Perez-Torres et al. (2008).

In the gamma-ray frequency range, these primary electrons emit via non-thermal bremsstrahlung and ICS against CMB radiation field if their energy spectrum, as we assume in this case, extends up to high energies (at least up to $E \sim 100-1000 \mathrm{GeV}$ in order to produce ICS emission in the energy range $0.1-10 \mathrm{GeV}$ ). Figure 2 shows that the EGRET upper limit on Ophiuchus, $F(>100 \mathrm{MeV}) \leq 5 \times 10^{-8} \mathrm{~cm}^{-2} \mathrm{~s}^{-1}$ (Reimer et al. 2003), is not exceeded in the $p=3.5$ case, while it is marginally exceeded in the $p=4.4$ case. In the first case, we can conclude that the HXR observation of the Ophiuchus cluster sets a constraint on the ICS emission from relativistic electrons that is stronger than the analogous limit set by EGRET; in the second case, the EGRET limit is stronger than the HXR limit. The signals we derive here for the gamma-ray emission of Ophiuchus in the PEM, and in particular the one derived from non-thermal bremsstrahlung emission, are sensibly larger than the Fermi sensitivity at $E \lesssim 300 \mathrm{MeV}$; therefore, this experiment could be able either to detect the bremsstrahlung gamma-ray emission from Ophiuchus or set even stronger limits on the non-thermal electron density.

We emphasize that the HXR data also sets indirectly a lower cut-off of $E_{\min } \sim 33$ and $90 \mathrm{MeV}$ for the two electron spectrum models considered in order to ensure that the heating rate does not exceeds the cooling rate.

\subsection{Secondary electron model from pp collisions (SEM-pp)}

To calculate the overall radiation emission from secondary electrons produced by collisions of cosmic ray (CR) non-thermal protons and the thermal protons of the IC gas (see Marchegiani et al. 2007 for details), we assume that the non-thermal protons have the following spectrum

$N_{\mathrm{p}}(E, r)=N_{\mathrm{p}, 0}(E / \mathrm{GeV})^{-s} g(r)$,

and we further assume, also in this case, that their spatial distribution is the same as that of the thermal IC gas out to the virial radius.

In a way similar to the PEM model, we assume proton spectral indices of $s=2.5$ and $s=3.4$, which again provide radio spectral indices of $\alpha_{\mathrm{R}}=1.25$ and 1.7, respectively.

The HXR data explained in terms of the ICS emission of the secondary electrons, provides values of $N_{p, 0}=$ $1.5 \times 10^{-6} \mathrm{GeV}^{-1} \mathrm{~cm}^{-3}$ (for $s=2.5$ ) and $N_{p, 0}=3.4 \times$ $10^{-5} \mathrm{GeV}^{-1} \mathrm{~cm}^{-3}$ (for $s=3.4$ ) for the normalization of the spectrum in Eq. (6).
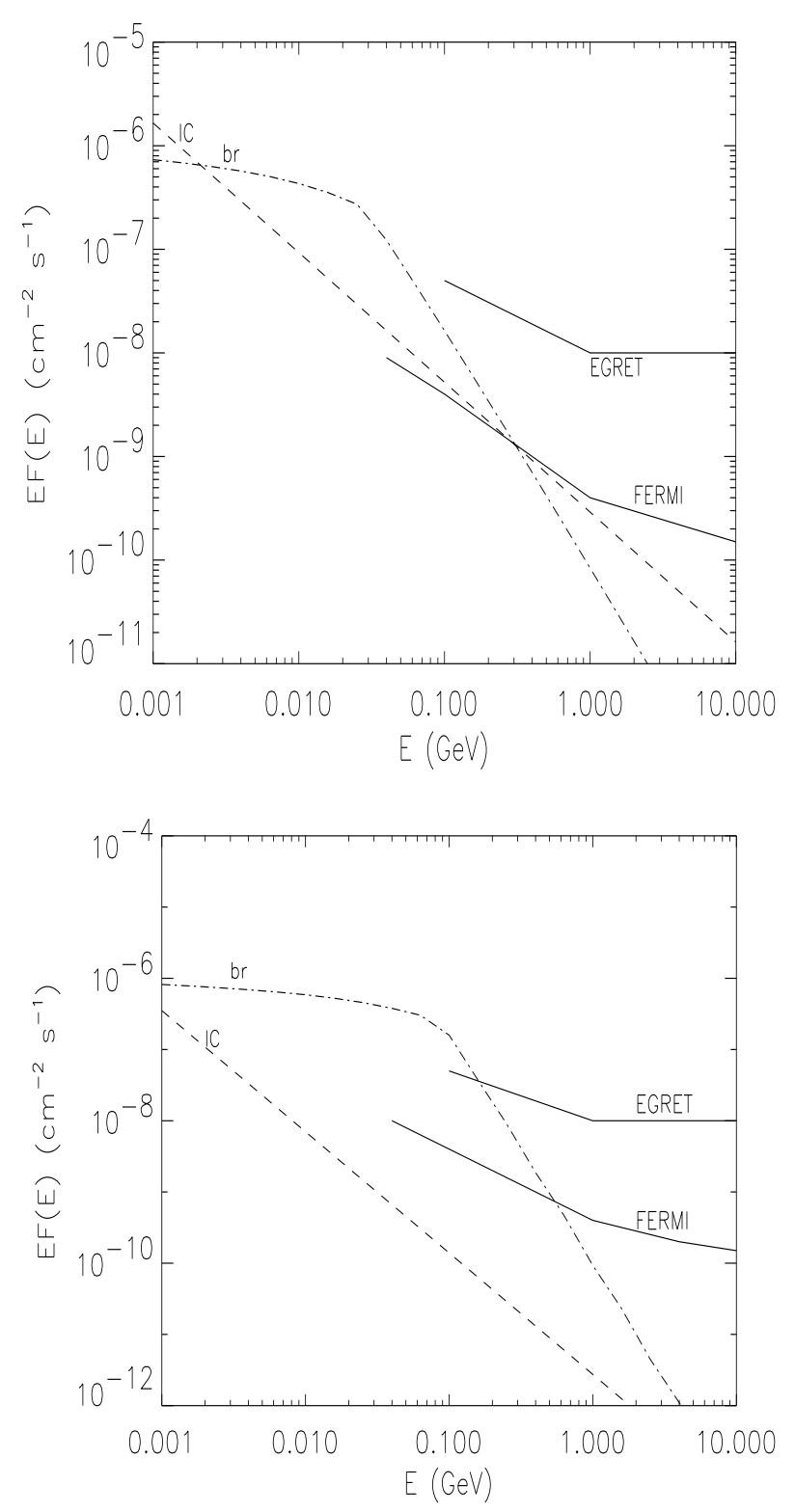

Fig. 2. The gamma-ray spectrum produced by primary electrons with $p=3.5$ (upper panel) and $p=4.4$ (lower panel) via ICS (dashed) and bremsstrahlung (dot-dashed) are compared to the sensitivity curves of EGRET and Fermi $(5 \sigma, 1$ year observation).

These values of the proton density normalization imply serious problems for Ophiuchus:

i) first, the proton pressure at the center of the cluster is more than 5 times higher than the thermal gas pressure for $s=$ 2.5 and 367 times higher for $s=3.4$, which causes serious problems for the cluster stability;

ii) secondly, the heating rate (Coulomb losses and hadronic collisions) induced by non-thermal protons in the cluster center is $\mathrm{d} \epsilon / \mathrm{d} t \sim 1.1 \times 10^{-25} \mathrm{erg} \mathrm{cm}^{-3} \mathrm{~s}^{-1}$ for $s=2.5$, and $\mathrm{d} \epsilon / \mathrm{d} t \sim 1.4 \times 10^{-24} \mathrm{erg} \mathrm{cm}^{-3} \mathrm{~s}^{-1}$ for $s=3.4$; these values are about 23 and 298 times higher than the cooling rate. This implies that there is a quite rapid heating of the cluster, $\mathrm{d}(k T) / \mathrm{d} t \sim 41 \mathrm{keV} \mathrm{Gyr}^{-1}$ and $\mathrm{d}(k T) / \mathrm{d} t \sim 531 \mathrm{keV} \mathrm{Gyr}^{-1}$, that will bring in a short time the whole IC gas to a temperature sensitively different (larger) from the observed one; 

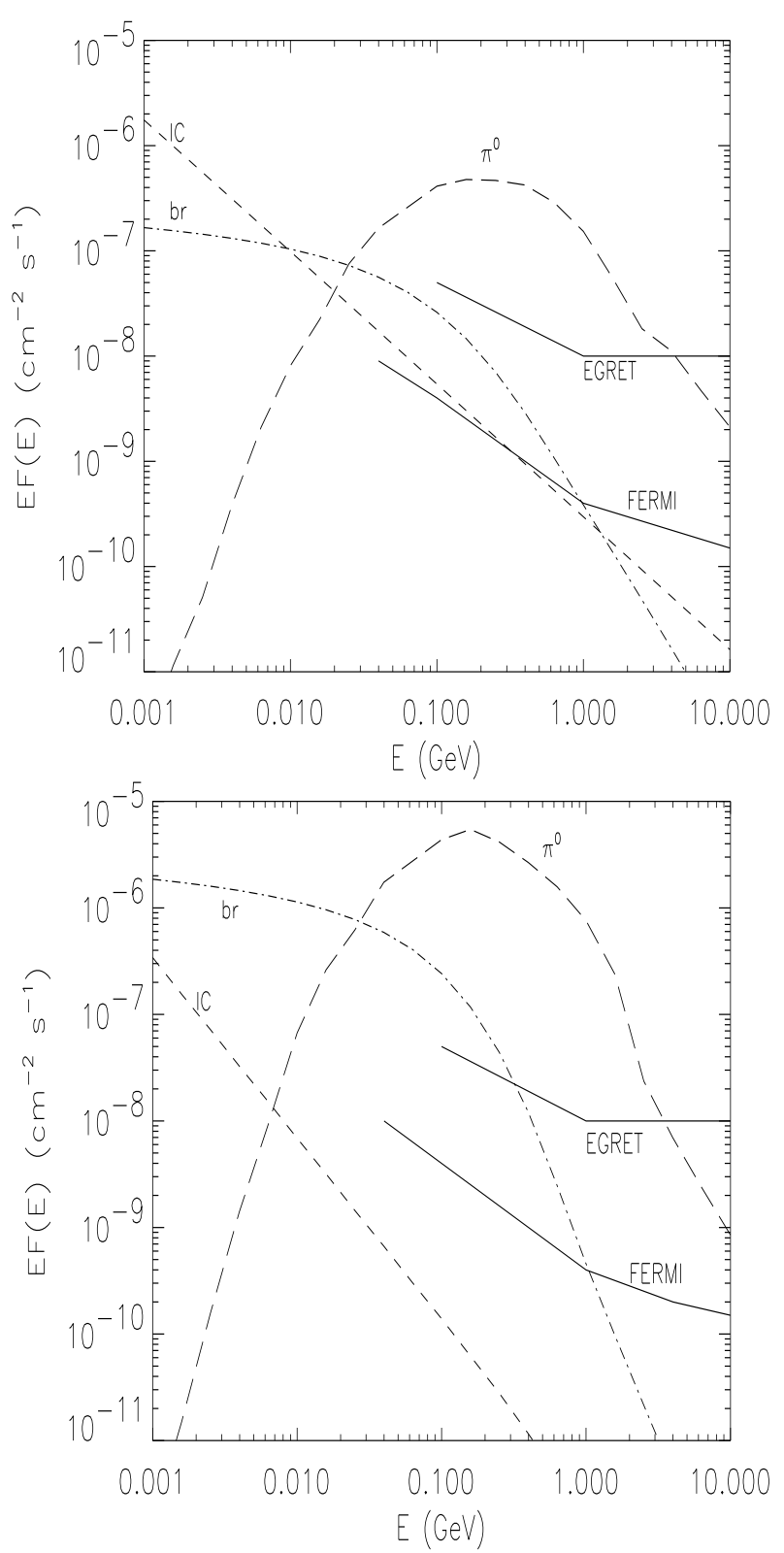

Fig. 3. The gamma-ray spectrum of Ophiuchus with $s=2.5$ (upper panel) and $s=3.4$ (lower panel) produced by ICS (dashes) and bremsstrahlung (dot dashes) of secondary electrons and by neutral pion decay (long dashes). We compare the predictions of the SEM-pp model with the sensitivity curves of EGRET and Fermi $(5 \sigma, 1$ year observation).

iii) finally, the gamma-ray emission produced by both secondary electrons and neutral pion decay (see Fig. 3) exceeds the EGRET upper limit for Ophiuchus by a factor $\sim 18$ and 170, for $s=2.5$ and 3.4 , respectively.

Thus, we must conclude that the HXR emission of Ophiuchus set by Swift and INTEGRAL, cannot be produced by secondary SEM-pp electrons. We note that this conclusion is analogous to that found for other clusters that we already studied such as Coma, A2199, A2163, and Perseus (Colafrancesco \& Marchegiani 2008).

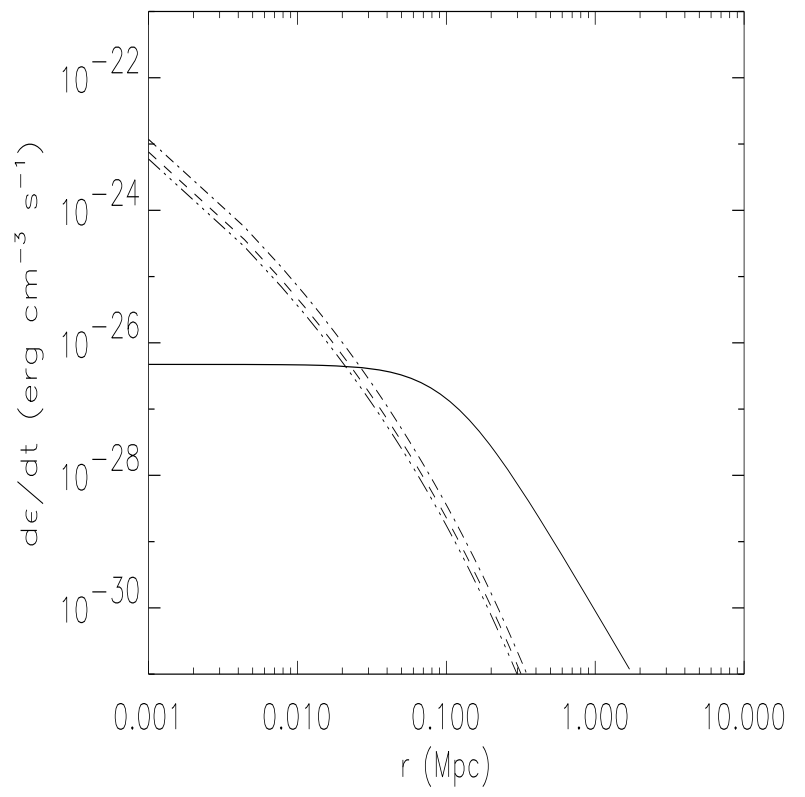

Fig. 4. The heating rate induced by secondary electrons produced by DM annihilation is shown at different radii for different neutralino models: $M_{\chi}=81 \mathrm{GeV}$ (dashed), $40 \mathrm{GeV}$ (dot-dashed), and $10 \mathrm{GeV}$ (dot-dot dashed). The intracluster gas cooling rate (solid) is also shown for comparison.

\subsection{Secondary electron model from $D M$ annihilation (SEM-DM)}

We consider here three neutralino DM models (similar to the analysis of Profumo 2008) with neutralino masses $M_{\chi}=$ $81\left(W^{+} W^{-}\right), 40(b \bar{b})$, and $10\left(\tau^{+} \tau^{-}\right) \mathrm{GeV}$.

For each neutralino model, we consider a radial DM density profile given by

$g_{\mathrm{DM}}(r)=\exp \left[-(2 / \alpha)\left(\left(r / r_{\mathrm{c}}\right)^{\alpha}-1\right)\right]$

(Navarro et al. 2004), where $\alpha=0.17$ and $r_{\mathrm{c}}$ equals to the core radius of the thermal gas density distribution. We assume that this DM radial profile extends out to the virial radius. The spectrum of the DM source function for the secondary electrons has, consequently, a radial distribution $\propto g_{\mathrm{DM}}^{2}(r)$.

To derive the equilibrium spectrum of these secondary electrons in Ophiuchus, we consider the role of the dominant energyloss mechanisms. These are ICS losses against CMB photons and synchrotron losses for electrons of energy higher than a few hundred $\mathrm{MeV}$ (note that synchrotron losses for magnetic fields $<3 \mu \mathrm{G}$, are negligible compared to the ICS losses), while at low energies $(\$ 150 \mathrm{MeV})$ the dominant energy loss mechanisms are Coulombian interactions with the IC gas particles. For this reason, the final spatial distribution of secondary electrons is proportional to $g_{\mathrm{DM}}^{2}(r)$ at high energies $(>150 \mathrm{MeV})$ and proportional to $g_{\mathrm{DM}}^{2}(r) / n_{\mathrm{th}}(r)$ at low energies $(<150 \mathrm{MeV})$.

The DM-produced secondary electron density is fixed, also in this case, by requiring that their ICS emission fits the observed HXR emission; this constraint corresponds to fixing the value of the neutralino annihilation cross-section, $\langle\sigma V\rangle$, because both the neutralino mass and its composition have been fixed by the chosen model.

This SEM-DM model also has serious implications for the Ophiuchus cluster:

i) the heating rate at the cluster center produced by the secondary SEM-DM electrons is very high; Fig. 4 shows the 


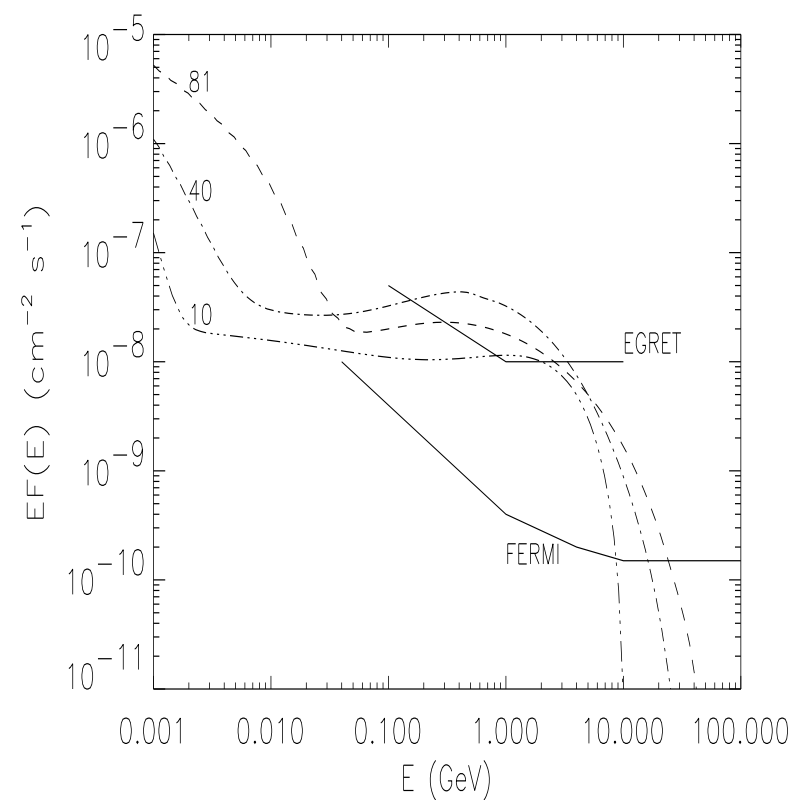

Fig. 5. The overall gamma-ray spectrum produced by the composition of the contributions of the secondary SEM-DM electrons (ICS and bremsstrahlung) and of the neutral pion decay for the three neutralino models here considered. The sensitivity curves of EGRET and Fermi $(5 \sigma, 1$ year observation) are shown for comparison.

secondary electrons heating rate at different radii compared to the cooling rate of the thermal IC gas. In fact, the heating rate largely exceeds the cooling rate in the cluster core at $r<30 \mathrm{kpc}$. This result implies a rapid over-heating of the Ophiuchus core, even though the volume integral of the heating rate is always lower than the volume integral of the cooling rate (this last quantity is $1.5 \times 10^{44} \mathrm{erg} \mathrm{s}^{-1}$, while the integrated heating rate is $4.6 \times 10^{42} \mathrm{erg} \mathrm{s}^{-1}, 7.4 \times$ $10^{42} \mathrm{erg} \mathrm{s}^{-1}$, and $3.6 \times 10^{42} \mathrm{erg} \mathrm{s}^{-1}$ for $M_{\chi}=81,40$, and $10 \mathrm{GeV}$, respectively);

ii) we show in Fig. 5 the gamma-ray emission spectra produced by the DM composite model worked out here via the three main mechanisms of gamma-ray emission: ICS and bremsstrahlung from secondary electrons, and neutral pion decay. All the three DM models considered in this composite DM model for Ophiuchus produce a gammaray flux that exceeds the EGRET limit, $F(>100 \mathrm{MeV})=$ $5.0 \times 10^{-8} \mathrm{~cm}^{-2} \mathrm{~s}^{-1}$ (the low-mass neutralino model with $M_{\chi}=10 \mathrm{GeV}$ is marginally consistent with the EGRET limit). The gamma-ray flux of Ophiuchus at $E>100 \mathrm{MeV}$ produced under the assumption that the same DM model reproduces the HXR data, are $7.4 \times 10^{-8}, 1.3 \times 10^{-7}$, and $4.3 \times 10^{-8} \mathrm{~cm}^{-2} \mathrm{~s}^{-1}$ for neutralino masses of 81,40 , and $10 \mathrm{GeV}$. A direct prediction of this DM model is that the gamma-ray flux produced by the three neutralino models considered here should be easily detectable by the Fermi experiment, whose results will be able, therefore, to validate or exclude this model for the origin of the HXR emission of Ophiuchus;

iii) Figure 6 shows the diffuse synchrotron radio spectra produced by the same secondary SEM-DM electrons by assuming a reference value of the average intracluster magnetic field in Ophiuchus of $0.1 \mu \mathrm{G}$. This figure shows that, for this value of magnetic field, a model with neutralino mass between 40 and $80 \mathrm{GeV}$ can reproduce the radio data.

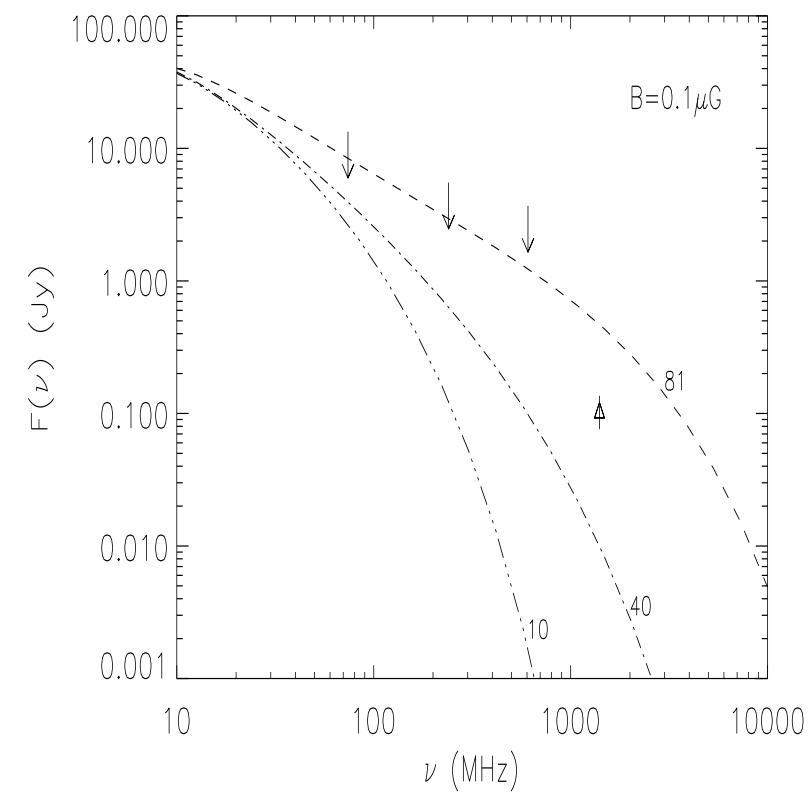

Fig. 6. The radio halo spectrum produced from secondary SEM-DM electrons via synchrotron emission in a constant magnetic field of $0.1 \mu \mathrm{G}$. Data are from Perez-Torres et al. (2008) (upper limits) and Govoni et al. (2009) (point at $1.4 \mathrm{GHz}$ ).

We also searched for the value of the magnetic field that, for each of the models we considered, reproduces the Ophiuchus radio halo flux at $1.4 \mathrm{GHz}$. Figure 7 shows the radio spectrum produced by SEM-DM electrons for best-fit magnetic field values of $0.055,0.18$, and $0.39 \mu \mathrm{G}$, for $M_{\chi}=81,40$, and $10 \mathrm{GeV}$, respectively. We can conclude that the $81 \mathrm{GeV}\left(W^{+} W^{-}\right)$model is consistent with radio data, while the $10 \mathrm{GeV}\left(\tau^{+} \tau^{-}\right)$model is not consistent. The $40 \mathrm{GeV}(b \bar{b})$ model is a border-line situation: we find, indeed, that for a slightly lower magnetic field of $0.17 \mu \mathrm{G}$, the radio spectrum is marginally consistent with the point at $1.4 \mathrm{GHz}$ and the upper limit at $74 \mathrm{MHz}$.

\section{Warming ray model}

We abandon now the strategy of fitting the ICS emission produced by high-E electrons to the HXR data of Swift and INTEGRAL because we evaluate - in the framework of the warming ray (WR) model (see Colafrancesco \& Marchegiani 2008; Colafrancesco et al. 2004) - the spectral and spatial characteristics of the WR proton population producing through their heating action the temperature structure of the Ophiuchus cluster, namely a constant temperature radial profile at the observed value of $k T \approx 9.9 \mathrm{keV}$.

The proton spectrum is written as in Eq. (6), with the values $s=2.5$ and $s=3.4$, and assuming a radial distribution given by $g(r) \propto g_{\mathrm{th}}^{\alpha}(r)$, where the value of $\alpha$ is found by fitting the radial temperature profile of the cluster (see Colafrancesco \& Marchegiani 2008, for technical details). The best fit analysis of the temperature profile of Ophiuchus provides the value $\alpha=1$, in analogy to what is found for other isothermal clusters (see discussion in Colafrancesco \& Marchegiani 2008), while the central WR density is $N_{\mathrm{p}, 0}=4.9 \times 10^{-8} \mathrm{~cm}^{-3} \mathrm{GeV}^{-1}$ and $N_{\mathrm{p}, 0}=9.4 \times 10^{-8} \mathrm{~cm}^{-3} \mathrm{GeV}^{-1}$ for $s=2.5$ and $s=3.4$ respectively, i.e., a factor $\approx 31$ and 362 lower than that required to reproduce, in this model, the Swift BAT/INTEGRAL HXR data. Consequently, the pressure ratio of the WR to the thermal gas at the cluster center is $P_{\mathrm{CR}} / P_{\text {th }} \sim 0.17$ for $s=2.5$ and 


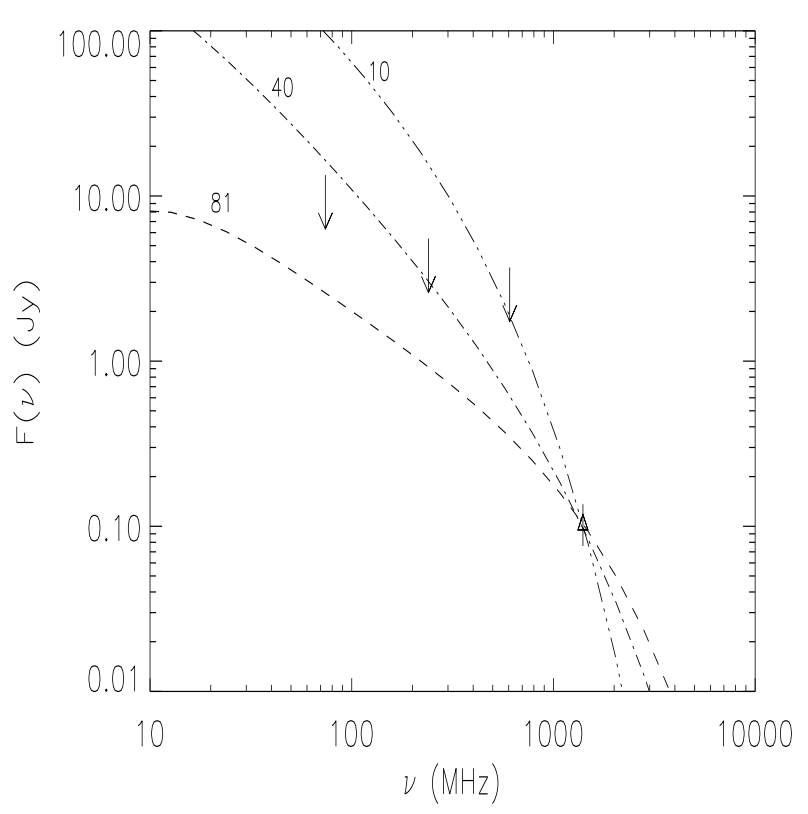

Fig. 7. The radio halo spectrum produced from secondary SEMDM electrons via synchrotron emission in a constant magnetic field of $0.055,0.18$, and $0.39 \mu \mathrm{G}$ for $M_{\chi}=81,40$, and $10 \mathrm{GeV}$, respectively. Data are from Perez-Torres et al. (2008) (upper limits) and Govoni et al. (2009) (point at $1.4 \mathrm{GHz}$ ).

$P_{\mathrm{CR}} / P_{\text {th }} \sim 1.0$ for $s=3.4$ : the first value does not affect the overall stability of the cluster in any way, while the second one is a problematic situation.

The diffuse radio emission produced by the secondary electron in this WR model is shown in Fig. 8 for various values of the uniform magnetic field; our results indicate that a uniform $B$-field of the order of $\sim 0.4 \mu \mathrm{G}$ is required to fit the available data for $s=2.5$, and $B \sim 2.0 \mu \mathrm{G}$ for $s=3.4$.

Figure 9 shows the diffuse gamma-ray emission from Ophiuchus as expected in the WR model: this emission consists of the combination of the neutral pion decay spectrum and ICS and bremsstrahlung emission from secondary electrons. The overall gamma-ray emission of Ophiuchus in the WR model, $F(>100 \mathrm{MeV})=3.3 \times 10^{-8} \mathrm{~cm}^{-2} \mathrm{~s}^{-1}$ and $2.3 \times 10^{-8} \mathrm{~cm}^{-2} \mathrm{~s}^{-1}$ for $s=2.5$ and $s=3.4$, respectively, is below the EGRET limit. However, the neutral pion decay gamma-ray emission predicted in this WR model should be detectable by Fermi with a $1 \mathrm{yr}$ observation ( $5 \sigma$ c.1.).

\section{Discussion and conclusions}

We have demonstrated that various and serious problems exist with the ICS interpretation of the HXR excess emission from the Ophiuchus cluster and, in general, of galaxy clusters for which an HXR emission excess has been detected. These problems are:

i) The true level of the HXR emission flux. The derivation of an HXR emission excess in clusters seems to depend strongly on the precise determination of the background thermal bremsstrahlung emission. Using INTEGRAL data, Eckert et al. (2008) derived an HXR flux in the 20-60 keV band of $F_{\mathrm{HXR}}=(10.1 \pm 2.5) \times 10^{-12} \mathrm{erg} \mathrm{cm}^{-2} \mathrm{~s}^{-1}$, for an IC gas temperature of $8.56_{-0.35}^{+0.37} \mathrm{keV}$. Using Swift BAT data, Ajello et al. (2009) derived an upper limit (90\% c.l.) of $F_{\text {HXR }} \leq 7.2 \times 10^{-12} \mathrm{erg} \mathrm{cm}^{-2} \mathrm{~s}^{-1}$, for a different IC gas temperature of $9.5_{-1.1}^{+1.4} \mathrm{keV}$; we note that the same authors (Ajello et al. 2009) derived an upper limit to the HXR flux of
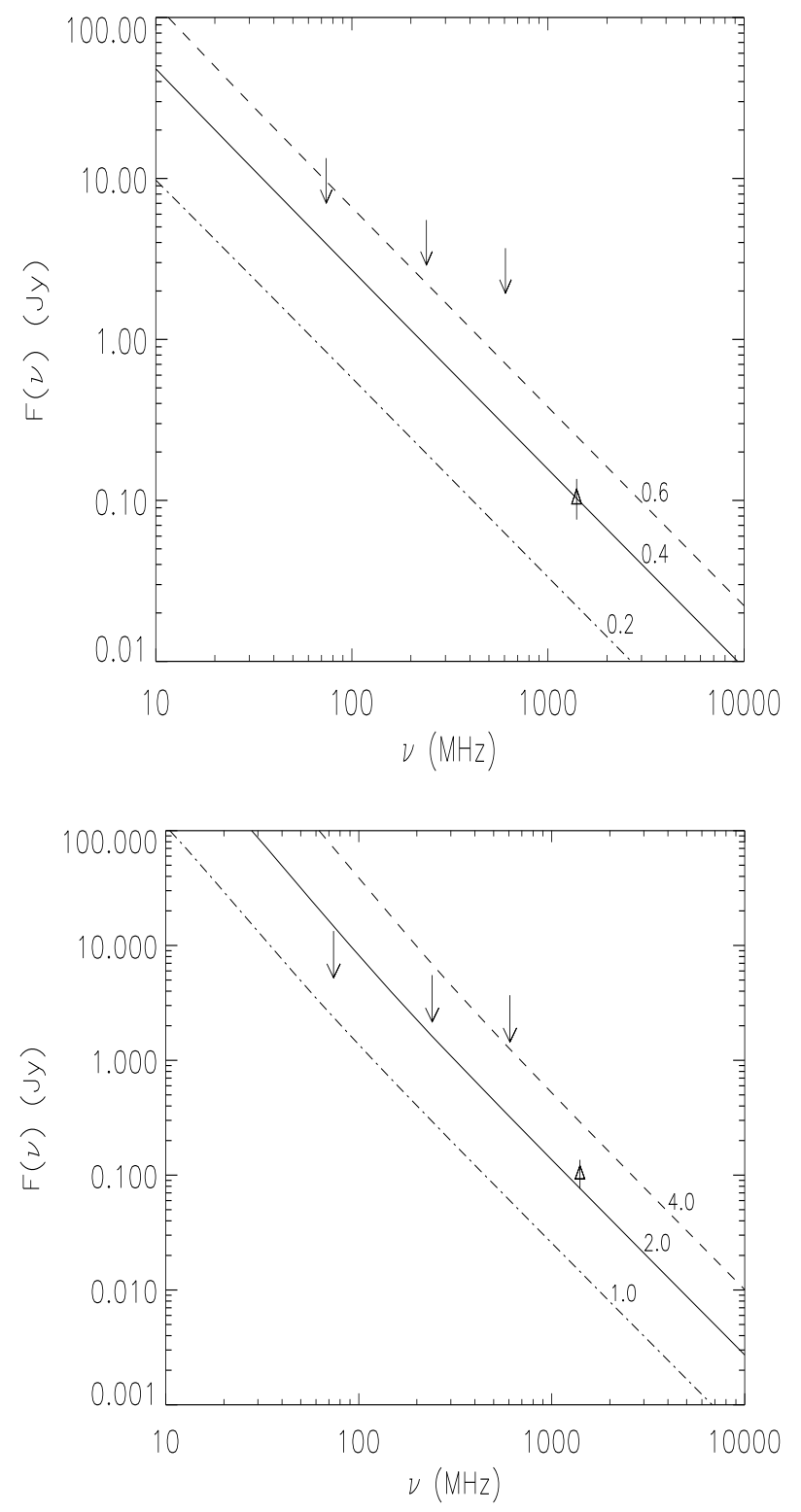

Fig. 8. The radio halo spectrum produced by secondary electrons with $s=2.5$ (upper panel) and $s=3.4$ (lower panel) in the WR model is shown for different values of a constant IC magnetic field (as labeled) in units of $\mu \mathrm{G}$. Data are from Perez-Torres et al. (2008) (upper limits) and Govoni et al. (2009) (point at $1.4 \mathrm{GHz}$ ).

$F_{\text {HXR }} \leq 4.5 \times 10^{-12} \mathrm{erg} \mathrm{cm}^{-2} \mathrm{~s}^{-1}$ by using a higher value of temperature $9.93_{-0.24}^{+0.24} \mathrm{keV}$, obtained by using a combination of Chandra and Swift-BAT data.

In conclusion, it is clear that a crucial input quantity in determining the value of the HXR excess flux is the detailed modeling of the thermal emission of the IC gas, because different values assumed for the IC gas temperature lead to different conclusions about the amount of HXR excess flux (see, e.g., the long standing discussion about evidence and counter evidence of the HXR excess in Coma, Fusco-Femiano et al. 1999, 2004, 2007, 2008; Rossetti \& Molendi 2004, 2007; see also Petrosian et al. 2008 for a review). For this reason, it would be extremely important to estimate the temperature of the IC gas with measurements that are independent of those obtained in the X-ray band. In this context, 

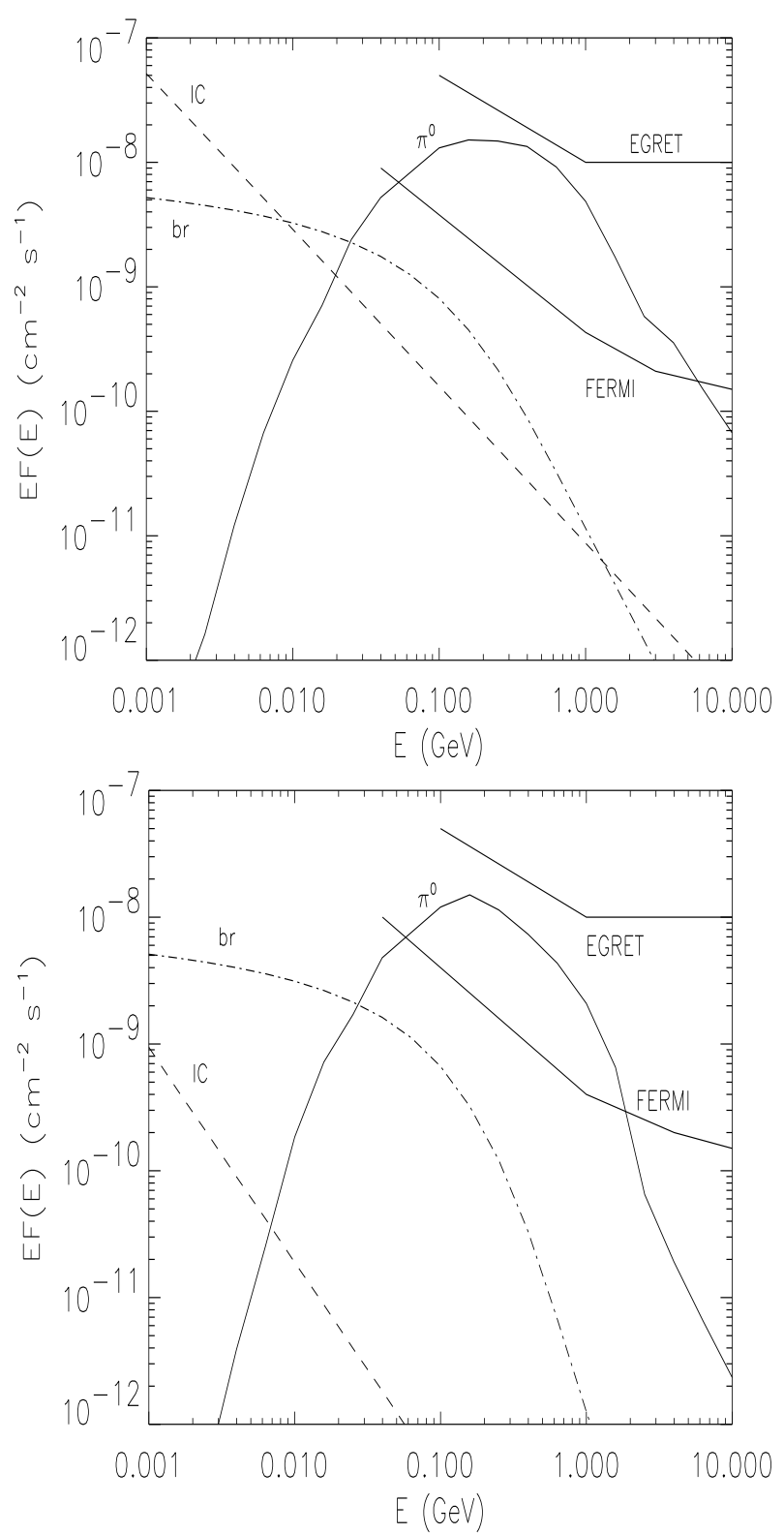

Fig. 9. The gamma-ray spectrum produced by secondary electrons with $s=2.5$ (upper panel) and $s=3.4$ (lower panel) via ICS (dashes) and bremsstrahlung (dot-dashes) and neutral pion decay (solid) in the WR model constrained to reproduce the observed temperature structures of the IC gas in Ophiuchus. The sensitivity curves of EGRET and Fermi ( $5 \sigma, 1$ year observation) are shown for comparison.

we note that a reliable method of measuring IC gas temperatures can be found by using Sunyaev-Zel'dovich observations over a wide spectral band (from $\sim 100$ to $\sim 400 \mathrm{GHz}$ ) reaching high frequencies where the sensitivity of the SZE to the cluster temperature is maximal (discussion of this issue in detail is found in a dedicated paper, Colafrancesco \& Marchegiani 2009; see also Colafrancesco et al. 2003; Colafrancesco 2007 for a review).

ii) The ICS HXR scenario. The hypothesis that high-E electrons are responsible for an ICS HXR emission at the level found by the combination of Swift-BAT and INTEGRAL observations leads to some important consequences.

First, to reconcile the HXR excess value with the relative diffuse synchrotron radio emission (from the same electron population) at the level observed in the same cluster, the value of the average magnetic field must be quite low and of the order of $\sim 0.1 \mu \mathrm{G}$ for $p=3.5$ and $\sim 0.2 \mu \mathrm{G}$ for $p=4.4$ (see Fig. 1). The result found for Ophiuchus is analogous to that derived for other clusters (see also our previous results discussed in Colafrancesco et al. 2005, Marchegiani et al. 2007). Specifically, we found that the data are consistent with an IC magnetic field of order $\sim 0.7$ and $1.2 \mu \mathrm{G}$ at the cluster center with a decreasing radial profile similar to that of the IC gas, for $p=3.5$ and 4.4 , respectively.

Secondly, there is a strong relation between the ICS HXR emission level and the relative gamma-ray emission and the consequences on the physics of the cluster, i.e., the heating of the IC gas and the ratio of non-thermal to thermal pressures:

- if the electrons producing the HXR emission are primaries, their gamma-ray emission (dominated at $E<1 \mathrm{GeV}$ by nonthermal bremsstrahlung) is slightly lower than the EGRET upper limit in the $p=3.5$ model, and slightly higher than this limit in the $p=4.4$ model, but certainly detectable by Fermi (see Fig. 2). If Fermi does not detect this gamma-ray emission, one should conclude that the ICS HXR emission is much lower than the Swift-INTEGRAL HXR detection and that the relativistic electron content of Ophiuchus is consequently much lower. The HXR data imply, in addition, a lower cut-off of $E_{\min } \sim 33$ and $93 \mathrm{MeV}$ (for $p=3.5$ and 4.4) on the electron spectrum in order to maintain a heating rate not higher than the cooling rate;

- if the electrons responsible for the ICS HXR emission are secondary particles produced in the decay of charged pions generated by cosmic-ray proton collisions with the IC gas protons (SEM-pp), then an ICS flux set at the HXR observations has unacceptable consequences. Specifically, we find that: in the $s=2.5$ case, the pressure exerted by relativistic protons at the cluster center is $\sim 5$ times higher than the thermal gas one; the heating rate induced by the same relativistic protons at the cluster center is $\sim 23$ times higher than the IC gas cooling rate; and the gamma-ray emission produced by neutral pion decay exceeds the EGRET limit by a factor $\sim 18$; in the $s=3.4$ case, these quantities increase to $\sim 367,298$, and 170, respectively (see Fig. 3). For all these reasons, we conclude that if electrons produce an ICS HXR emission in the observed range, they cannot be secondary (in the SEM-pp). This conclusion is analogous to that found for other clusters like Coma, A2199, A2163, and Perseus (see Colafrancesco \& Marchegiani 2008);

- if electrons are produced by neutralino DM annihilation, we have found that: the heating rate they induce at the cluster center is quite high (see Fig. 4); the relative gamma-ray emission exceeds the EGRET limit for the two high- $M_{\chi}$ models considered here $\left(M_{\chi}=40\right.$ and $\left.81 \mathrm{GeV}\right)$ (see Fig. 5), with a marginal consistency for the low-mass model with $M_{\chi}=10 \mathrm{GeV}$; the radio flux produced by electrons is consistent with the available data for $M_{\chi}>40 \mathrm{GeV}$, and for $B<0.18 \mu \mathrm{G}$. Therefore, the information inferred by gamma and radio data are not compatible, and we conclude that it is not possible to conceive that the ICS HXR emission of secondary SEM-DM electrons has a flux close to that of the available observations (i.e., the maximum allowed flux set by Swift and INTEGRAL, see Sect. 1), and thus their annihilation cross-section must be much lower than the values used by Profumo (2008). Even normalizing these models to the lower allowed flux value of the HXR excess of Ophiuchus (see our discussion in Sect. 1), all the previous 
results vary (decrease) by $\sim 15 \%$, leaving our basic conclusions unchanged.

iii) Relaxing the assumption of recovering the observed HXR excess and assuming that non-thermal protons act as warming rays (see Colafrancesco \& Marchegiani 2008), it is possible to paint a much more acceptable scenario in which the unacceptable pressure ratios derived in SEM models do not hold since the ratio $P_{\text {non-th }} / P_{\text {th }} \approx 0.17$ and 1.0 for $s=2.5$ and 3.4, respectively, and is constant throughout the cluster (this is because non-thermal protons must have the same spatial distribution as the thermal IC gas to recover the spatial temperature distribution of the cluster).

In addition, the WR model has other positive aspects for the cluster structure:

i) it does not induce excess heating effects, since a quasistationary balance between heating and cooling is the working assumption of the WR model;

ii) we found that the diffuse radio emission produced in this case requires, for $s=2.5$ and $s=3.4$ respectively, a value of the average magnetic field of $\sim 0.4$ and $2 \mu \mathrm{G}$ (see Fig. 8) and a central value of $\sim 1.1$ and $6 \mu \mathrm{G}$ with a radial profile similar to that of the IC gas, which is consistent with the general findings for clusters with Faraday Rotation measurements (see, e.g., Carilli \& Taylor 2002; Govoni \& Feretti 2004);

iii) the gamma-ray emission produced in this model is considerably lower than the EGRET limit but definitely detectable by Fermi (see Fig. 9). The Fermi detection of this gammaray emission from Ophiuchus will have a crucial impact in proving or disproving this model.

In this WR model, the HXR ICS flux of Ophiuchus is much lower (by a factor $\sim 30$ and 362 for $s=2.5$ and $s=3.4$, respectively) than the limit set by the present observations (by INTEGRAL and Swift-BAT) and could only be detectable by using long-exposure observations with the next generation HXR instruments like NeXT (see e.g., Takahashi et al. 2004).

To conclude, models of high-E electrons in clusters that can be adjusted to reproduce their ICS HXR emission at the level indicated by the available observations fail to work because they would imply unacceptable levels of heating and gamma-ray emission. In contrast, models of high-energy particles that are able to reproduce the IC gas temperature structure (i.e., the WR model) predict a level of non-thermal HXR ICS emission that is far below the current limits obtained with INTEGRAL and Swift-BAT, and provide an overall Spectral Energy Distribution that is consistent with all the available data - from radio to gamma-rays - for Ophiuchus as well as for other clusters.

Acknowledgements. We thank P. Ullio for providing detailed source spectra of the neutralino annihilation models considered in this paper. We also thank the referee, M. A. Perez-Torres, for his comments and suggestions that allowed us to improve the presentation of our results.

\section{References}

Ajello, M., Rebusco, P., Cappelluti, N., et al. 2009, ApJ, 690, 367 Atoyan, A., \& Volk, H. 2000, ApJ, 535, 45

Blasi, P., \& Colafrancesco, S. 1999, Astropart. Phys., 12, 169 Brunetti, G. 2004, JKAS, 37, 493

Carilli, C. L., \& Taylor, G. B. 2002, ARA\&A, 40, 319

Colafrancesco, S. 2007, New Astron. Rev., 51, 394

Colafrancesco, S., \& Blasi, P. 1998, Astropart. Phys., 9, 227

Colafrancesco, S., \& Marchegiani, P. 2008, A\&A, 484, 51

Colafrancesco, S., \& Marchegiani, P. 2009, preprint

Colafrancesco, S., Marchegiani, P., \& Palladino, E. 2003, A\&A, 397, 27

Colafrancesco, S., Dar, A., \& De Rújula, A. 2004, A\&A, 413, 441

Colafrancesco, S., Marchegiani, P., \& Perola, G. C. 2005, A\&A, 443, 1

Colafrancesco, S., profumo, S., \& Ullio, P. 2006, A\&A, 455, 21

Dogiel, V. A., Colafrancesco, S., Ko, C. M., et al. 2007, A\&A, 461, 433

Eckert, D., Produit, N., Paltani, S., Neronov, A., \& Courvoisier, T. J.-L. 2008, A\&A, 479, 27

Ensslin, T., \& Biermann, P. 1998, A\&A, 330, 90

Fujita, Y., Hayashida, K., Nagai, M., et al. 2008, PASJ, 60, 1133

Fusco-Femiano, R., dal Fiume, D., Feretti, L., et al. 1999, ApJ, 513, L21

Fusco-Femiano, R., Orlandini, M., Brunetti, G., et al. 2004, ApJ, 602, L73

Fusco-Femiano, R., Landi, R., \& Orlandini, M. 2007

[arXiv:astro-ph/0702576]

Fusco-Femiano, R., \& Orlandini M. 2008 [arXiv:0802 . 1817]

Govoni, F., \& Feretti, L. 2004, Inter. J. Mod. Phys. D, 13, 1549

Govoni, F., Murgia, M., Markevitch, M., et al. 2009, A\&A, 499, 371

Inoue, S., Aharonian, F. A., \& Sugiyama, N. 2005, ApJ, 628, L9

Johnston, M. D., Bradt, H. V., Doxsey, R. E., et al. 1981, ApJ, 245, 799

Marchegiani, P., Perola, G. C., \& Colafrancesco, S. 2007, A\&A, 465, 41

Mohr, J. J., Mathiesen, B., \& Evrard, A. E. 1999, ApJ, 517, 627

Navarro, J. F., Hayashi, E., \& Power, C. 2004, MNRAS, 349, 1039

Nevalainen, J., Oosterbroek, T., Bonamente, M., \& Colafrancesco, S. 2004, ApJ, 608,166

Perez-Torres, M. A., Alberdi, A., Romero-Canizales, C., et al. 2008 [arXiv: 0812.3598]

Petrosian, V., Bykov, A., \& Rephaeli, Y. 2008, Space Sci. Rev., 134, 191

Profumo, S. 2008, AIP Conf. Proc., 1078, 551

Reimer, O., Pohl, M., Sreekumar, P., \& Mattox, J. R. 2003, ApJ, 588, 155

Rossetti, M., \& Molendi, S. 2004, A\&A, 414, L41

Rossetti, M., \& Molendi, S. 2007 [arXiv: astro-ph/0702417]

Sarazin, C. 1999, ApJ, 520, 529

Slee, O. B. 1977, AuJPA, 43, 1

Slee, O. B., \& Higgins, C. S. 1975, AuJPA, 36, 1

Takahashi, T., Makishima, K., Fukazawa, Y., et al. 2004, New AR, 48, 269

Timokhin, A. N., Aharonian, F. A., \& Neronov, A. Yu. 2004, A\&A, 417, 391

Watanabe, M., Yamashita, K., Furuzawa, A., Kunieda, H., \& Tawara, Y. 2001, PASJ, 53605 